\section{Applicability and validity of the Amnestic Comparative Self-Assessment in adolescents}

\author{
Karolin Roeser, ${ }^{1}$ Barbara Schwerdtle, 1 \\ Ruth Eichholz, ${ }^{1}$ Andrea Kübler ${ }^{1,2}$ \\ 1Department of Psychology I, University \\ of Würzburg; 2Institute of Medical \\ Psychology and Behavioral Neurobiology, \\ University of Tübingen, Germany
}

\begin{abstract}
The Amnestic Comparative Self-Assessment (ACSA) is a sensitive, efficient, and economic instrument to assess overall quality of life in adult populations. The present study investigates the applicability of the ACSA in an adolescent sample and compares it to a measure of health-related quality of life, the KiddoKindl. The sample comprised 92 adolescents (50 girls, 42 boys) aged 11-17 years (mean age: 13.67, standard deviation: 1.34). Of the investigated sample, $\mathrm{n}=69$ (75\%) completed the ACSA. No significant demographic differences were found between ACSA-respondents and non-respondents. The correlation of the KiddoKindl and the ACSA was moderate $(r=0.50)$. The Kiddo-Kindl subscales and the ACSA correlated between $r=0.07$ and 0.41 . The majority of adolescents are able to complete the ASCA, and its acceptance and validity are independent of age. Thus, future investigations could adopt the ACSA in adolescents to assess overall quality of life.
\end{abstract}

\section{Introduction}

The concept of quality of life [quality of life $(\mathrm{Q} O \mathrm{~L})]$ was established in the field of medicine in the 80's. ${ }^{1}$ Until today, QoL has become a major outcome measure in clinical investigations. ${ }^{2}$ However, a universally approved definition of QoL is lacking. An established approach to QoL was formulated by Bernheim, who defined QoL as an individual and emergent construct, the resultant of a great many interactions, and of a different order than its contributing components. 3 Therefore, the assessment of overall QoL has to allow individuals to use their own internal standards and priorities as references. According to this definition, the Amnestic Comparative Self-Assessment [Amnestic Comparative Self-Assessment (ACSA)] uses a one-dimensional, self-anchoring scale. ${ }^{4}$ Its endpoints refer to the subject's best and worst times in life and his/her actual overall well-being is rated between these inter- nal anchors. The ACSA has been employed in various adult samples, for example cancer patients, ${ }^{5}$ locked-in-syndrome patients, ${ }^{6}$ or organ donors. ${ }^{7}$ It has proven to be a sensitive measure, possessing greater sensitivity than conventional single-item measures of life satisfaction and happiness. ${ }^{8,9}$

However, in children and adolescents, the ACSA has to our knowledge not yet been adopted. This may be because self-anchoring scales, like the ACSA, increase processing time and cause more drop-outs as compared to fixedanchoring scales, ${ }^{10}$ or because completing the ACSA requires that a person has life experience, which sets the standard of comparison. ${ }^{11}$ On the other hand, its use would enrich future epidemiological or clinical investigations, because it is a sensitive, efficient and economic instrument. Developmental processes in the course of adolescence strengthen the ability for abstract thinking and metacognitions. ${ }^{12,13}$ With increasing age, also the stability of the self-concept increases. ${ }^{14}$ Thus, adolescents may well be able to specify their overall QoL and complete the ACSA. In future research, the ACSA could be used as an alternative or in addition to the health-related approach to QoL, which is at present predominant in children and adolescents. ${ }^{15}$

Health-related quality of life [Health-related quality of life (HRQoL)] is defined as a multidimensional construct pertaining to the physical, emotional, mental, social and behavioral components of wellbeing and function as perceived by the patients andor observers. ${ }^{16}$ It is measured by evaluating satisfaction with different domains of the construct. 17 Thus, the assessment of HRQoL includes ratings on distinct subscales, which are considered relevant to QoL for the majority of a cultural group. However, we cannot know to what extent the HRQOL dimensions and their interactions contribute to an individual's overall QoL, because the weights of the distinct dimensions remain unknown and, therefore, immeasurable. ${ }^{3}$ By contrast, the ACSA bypasses biases due to cultural differences, peer-relativity, and social desirability, because individuals are required to integrate the multiple facets of QoL based on their personal life-experience. Peer-relativity and social desirability might affect QoLmeasures that do not use internal standards as references especially in adolescents because, in this age-group, relations to others are of major importance. ${ }^{12}$ Therefore, an instrument like the ACSA, which is not susceptible to external influences, would be of special value in adolescent samples. Due to its independence of cultural and social influences, the ACSA could be adopted in cross-cultural studies or for comparisons between adolescent populations who might differ in their external standards. We therefore consider the ACSA a convenient instrument, whose implementa-
Correspondence: Karolin Roeser, Department of Psychology I, University of Würzburg, Marcusstraße 9-11, D-97070, Würzburg, Germany.

Tel. +49.931 .3181012 - Fax: +49.931 .3182424$

E-mail: karolin.roeser@uni-wuerzburg.de

Key words: adolescence, quality of life, amnestic comparative self-assessment, health-related quality of life.

Contributions: KR has made substantial contributions to the conception of the study, carried out statistical analysis and drafted the manuscript; BS participated in designing the study and revised the manuscript critically; RE collected data, helped to perform statistical analysis and participated in the interpretation of data; AK has been involved in designing the study and in drafting the manuscript and revised it critically.

Conflict of interests: the authors declare no potential conflict of interests.

Received for publication: 18 0ctober 2012. Revision received: 18 December 2012. Accepted for publication: 4 January 2013.

This work is licensed under a Creative Commons Attribution NonCommercial 3.0 License (CC BYNC 3.0).

(C) Copyright K. Roeser et al., 2013

Licensee PAGEPress, Italy

Health Psychology Research 2013; 1:e8

doi:10.4082/hpr.2013.e8

tion could make a valuable contribution to $\mathrm{QoL}$ research in adolescents.

The aim of the present study was to investigate the usability of the ACSA in adolescents and compare results to that of the Kiddo-Kindl, a validated and widely-used instrument specifically developed for 12 - to 16 -year-olds. ${ }^{18,19} \mathrm{We}$ expected a moderately positive correlation between the two measures. This correlation should be consistently high in all age groups of the adolescent sample. According to the findings of van Acker and Theuns, 10 who reported more drop-outs in self- as compared to fixedanchoring scales, we expected responds rates to the ACSA to be lower than those to the HRQoL-measure. ACSA-respondents and nonrespondents were analyzed for systematic differences between each other in sex, age, educational level, and HRQoL.

\section{Materials and Methods}

The here presented data were collected in the context of a study about the association of sleep problems and HRQoL in German adolescents. ${ }^{20}$ Study materials consisted of a covering 
letter, a letter of agreement, questionnaires (see below), and the assessment of demographic variables (including age, sex, and type of school). Documents were distributed in schools, youth centers, and sports clubs by the experimenters. The parents of all participants gave written informed consent prior to taking part in the study. The study was conducted in accordance with our institution's ethical review committee and the standard ethical guidelines as defined by the Declaration of Helsinki (World Medical Association).

Amnestic Comparative Self-Assessment: ${ }^{4}$ The ACSA consists of one single item. Subjects are instructed to memorize the best and worst times in their lives and rate their actual overall well-being on an ordinal visual analog scale ranging from -5 to +5 in relation to their individual anchors.

Kiddo-Kindl: 19 The Kiddo-Kindl questionnaire was constructed for children aged 12-16 years. It consists of 30 items assessing HRQoL on six subscales (physical well-being, emotional well-being, self-esteem, family, friends, and everyday functioning/school). Transformed scores for the total score and each subscale can be derived ranging from 0 to 100 on an interval scale. The empirical evaluation of the KiddoKindl has shown good reliability, validity, and acceptance in adolescents. ${ }^{19}$

All analyses were conducted with SPSS Statistics 18 (IBM Deutschland $\mathrm{GmbH}$, Ehningen). Independent samples $t$-tests and chi-square tests were conducted to compare ACSA-respondents and non-respondents. Since the ASCA is answered on an ordinal scale, reported correlations are Spearman-Rho coefficients or Spearman partial correlation, indicating convergent validity. Analyzing divergent validity, group differences between boys and girls were tested using independent samples $t$ test for the Kiddo-Kindl, and the non-parametric Mann-Whitney-U-test for differences in ACSA ratings.

\section{Results}

The sample comprised 92 adolescents (50 girls, 42 boys) aged 11-17 years ( $M=13.67$, $\mathrm{SD}=1.34)$. Of the sample, $\mathrm{n}=69$ participants (75\%) completed the ASCA. ACSA-respondents and non-respondents did not differ significantly in age, sex, type of school or Kiddo-Kindl total score (Table 1). Frequencies of ACSA score (Figure 1) show a left skewed, platykurtic distribution (skewness: -0.76, excess kurtosis: $-2.68)$.

The correlation of the Kiddo-Kindl total score $(\mathrm{M}=70.78, \mathrm{SD}=11.07)$ and the ACSA rating $(\mathrm{MD}=2.00$, Range $=10)$ was medium $(r=0.50)$. The correlation of the Kiddo-Kindl total score and the ACSA was still medium when controlled for age (partial $r=0.53$ ). As depicted in Table 2, correlations of Kindl subscales and the ACSA varied between $r=0.07$, $n s$ (everyday functioning/school) and $r=0.41, \mathrm{P}<0.01$ (emotional well-being). Again, controlling for age did not significantly affect the correlation coefficients. In ACSA-respondents, Kiddo-Kindl total score differed significantly between boys $(\mathrm{M}=74.02$, $\mathrm{SD}=9.19)$ and girls $(\mathrm{M}=68.99, \mathrm{SD}=11.78$, $\left.t_{(67)}=-1.97, \mathrm{P}=0.05\right)$. Correspondingly, boys (mean rank: 39.51) also reported higher ACSA scores as compared to girls (mean rank: 30.61 , Mann-Whitney- $\mathrm{U}=441.50, \mathrm{P}=0.06$ ).

\section{Discussion}

The aim of the present study was to investigate the applicability of the ACSA in adolescents and to compare it to the Kiddo-Kindl, an established and well-evaluated measure of HRQoL. We could confirm our first hypothesis of a positive relationship between overall and health-related QoL: the correlation between the ACSA and the Kiddo-Kindl score was moderate and positive. This moderate correlation indicates common aspects captured by both measures, such as emotional and physical wellbeing, but also indicates differences, which are specifically obvious for the Kiddo-Kindl sub-

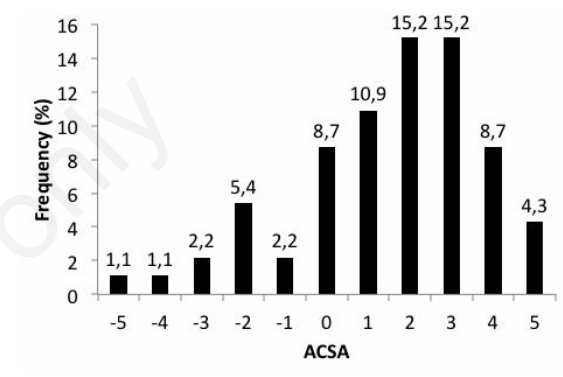

Figure 1. Answer frequencies for Amnestic comparative self-assessment in percent.

Table 1. Impacts of demographic variables on Amnestic Comparative Self-Assessmentresponse.

\begin{tabular}{|c|c|c|c|}
\hline & $\begin{array}{l}\text { ACSA } \\
\text { respondents }\end{array}$ & $\begin{array}{l}\text { ACSA } \\
\text { non-respondents }\end{array}$ & Test statistics \\
\hline Age (years) & $\mathrm{M}=13.68(\mathrm{SD}=1.30)$ & $\mathrm{M}=13.65(\mathrm{SD}=1.50)$ & $t_{(90)}=-0.09, \mathrm{~ns}$. \\
\hline $\begin{array}{l}\text { Sex } \\
\text { Girls } \\
\text { Boys }\end{array}$ & $\begin{array}{l}\mathrm{n}=35(70.00 \%) \\
\mathrm{n}=34(80.95 \%)\end{array}$ & $\begin{array}{l}\mathrm{n}=15(30.00 \%) \\
\mathrm{n}=8(19.05 \%)\end{array}$ & $X_{(1)}=1.46, \mathrm{~ns}$. \\
\hline $\begin{array}{l}\text { Type of school } \\
\text { Gesamtschule* } \\
\text { Gymnasium }^{\circ}\end{array}$ & $\begin{array}{l}\mathrm{n}=38(69.09 \%) \\
\mathrm{n}=31(83.78 \%)\end{array}$ & $\begin{array}{l}\mathrm{n}=17(30.91 \%) \\
\mathrm{n}=6(16.22 \%)\end{array}$ & $X_{(1)}=2.55, \mathrm{~ns}$. \\
\hline Kiddo-Kindl & $\mathrm{M}=71.47(\mathrm{SD}=0.81)$ & $\mathrm{M}=68.30(\mathrm{SD}=11.74)$ & $t_{(90)}=-1.19, \mathrm{~ns}$ \\
\hline
\end{tabular}

ACSA, Amnestic Comparative Self-Assessment; M, mean; SD, standard deviation; ns, not significant. *Equivalent to comprehensive school; equivalent to grammar/prep school.

Table 2. Correlations of Kindl subscales and Amnestic Comparative Self-Assessment..

\begin{tabular}{lll} 
& \multicolumn{1}{c}{ Amnestic comparative self-assessment } \\
& $r$ & $\begin{array}{c}\text { partial } r \\
\text { (partial correlation controlled for age) }\end{array}$ \\
Physical well-being & $0.32^{*}$ & $0.34^{*}$ \\
Emotional well-being & $0.41^{*}$ & $0.42^{*}$ \\
\hline Self-esteem & $0.33^{*}$ & $0.36^{*}$ \\
Family & $0.40^{*}$ & $0.40^{*}$ \\
\hline Friends & $0.33^{*}$ & $0.34^{*}$ \\
Everyday functioning & 0.07 & 0.09 \\
\hline Total & $0.50^{*}$ & $0.53^{*}$
\end{tabular}

${ }^{* \mathrm{P}<0.01 \text {. }}$ 
the ACSA. Five participants stated Stupid question, I don't think and feel in those categories or ?. But the reduced response rate cannot be considered a problem exclusively occurring in adolescents. Self-anchoring scales, like the ACSA, cause comparably elevated drop-out rates in adults (26.98\%).20 Thus, not only adolescents, but also adults have reduced response rates as compared to fixed-anchoring scales. ACSArespondents and non-respondents did not differ in demographic variables or HRQoL. Therefore, acceptance of the ACSA did not depend on individual characteristics; not even on the participants' age. This is remarkable, because the study included adolescents from a broad age range (11-17 years), who were equally able to complete the ACSA.

However, several limitations to the validity of our results have to be considered. Firstly, the study material was not assessed in a standardized environment. Biases due to distractors or parental influence on adolescents' answers cannot be ruled out. Secondly, our sample was recruited in a large city and might not be representative for adolescents from less urbanized regions. Thirdly, the recruitment via public institutions might have caused a selection bias. Education and social integration are likely to be above average in our sample. Future investigations in larger representative samples would be desirable to further explore convergent and divergent validity of the ACSA in adolescents. Future research might also address the question of how completion rates could be increased. In this regard, the immediate presence of an experimenter or a modified, agespecific instruction may prove helpful.

To conclude, the ACSA is applicable in the majority of adolescents and can be used in future investigations to assess overall QoL, for example in epidemiological and cross-cultural surveys, clinical populations or as an outcome measure in intervention studies. Overall QoL correlates moderately positive with HRQoL and less positively with separate HRQoL scales. The ACSA is as well accepted in adolescents as other self-anchoring scales in adults, and acceptance and validity do not depend on age or other individual characteristics.

\section{References}

1. Najman JM, Levine S. Evaluating the impact of medical care and technologies on the quality of life: A review and a critique. Soc Sci Med 1981;15:107-15.

2. Kowalski C, Pennell S, Vinokur A. Felicitometry: measuring the quality in quality of life. Bioethics 2008;22:307-13.

3. Bernheim JL. How to get serious answers to the serious question: how have you been?: subjective quality of life (QOL) as an individual experiential emergent construct. Bioethics 1999;13:272-87.

4. Bernheim J, Buyse M. The amnestic comparative self-assessment for measuring the subjective quality of life in cancer patients. J Psychosoc Oncol 1993;1:25-38.

5. van Knippenberg FC, de Haes JC. Measuring the quality of life of cancer patients: psychometric properties of instruments. J Clin Epidemiol 1988;41: 1043-53.

6. Bruno MA, Bernheim JL, Ledoux D, et al. A survey on self-assessed well-being in a cohort of chronic locked-in syndrome patients: happy majority, miserable minority. BMJ Open 2011;1:e000039.

7. Walter M, Bronner E, Pascher A, et al. Psychosocial outcome of living donors after living donor liver transplantation: a pilot study. Clin Transplant 2002;16:33944.

8. Moller V, Theuns P, Erstad I, Bernheim J. The best and worst times of life: Narratives and assessments of subjective well-being by Anamnestic Comparative Self Assessment (ACSA) in the Eastern Cape, South Africa. Soc Indic Res 2008; 89:1-22.

9. Bernheim J, Theuns P, Mazaheri M, et al. The potential of Anamnestic Comparative Self-Assessment (ACSA) to reduce bias in the measurement of subjective well-being. J Happiness Stud 2006;7:227-50.

10. Van Acker F, Theuns P. On possibilities and limitations of using self-anchoring scales in web surveys. Qual Quant 2010;44:112937.

11. Heylighen F, Bernheim J. Measuring global progress through subjective well-being. Proceedings of the III Conference of the ISQ0LS; 2001: University of Girona Press.

12. Christie D, Viner R. Adolescent development. BMJ 2005;330:301-4.

13. Schneider W. The development of metacognitive knowledge in children and adolescents: Major trends and implications for education. Mind Brain Educ 2008;2:114-21.

14. Alsaker FD, Olweus D. Stability of global self-evaluations in early adolescence: A cohort longitudinal study. J Res Adolesc 1992;2:123-45.

15. Harding L. Children's quality of life assessments: a review of generic and health related quality of life measures completed by children and adolescents. Clin Psychol Psychot 2001;8:79-96.

16. Bullinger M. Quality of life - definition, conzeptualization and implications: a methodologist's view. Theor Surg 1991;6: 143-9.

17. Solans M, Pane S, Estrada MD, et al. Health-related quality of life measurement in children and adolescents: a systematic review of generic and disease-specific instruments. Value in Health 2008;11:74264.

18. Ravens-Sieberer U, Bullinger M. Assessing health-related quality of life in chronically ill children with the German KINDL: first psychometric and content analytical results. Qual Life Res 1998;7: 399-407.

19. Ravens-Sieberer U, Bullinger M. News from the KINDL-Questionnaire - A new version for adolescents. Qual Life Res 1998;7:653.

20. Roeser K, Eichholz R, Schwerdtle B, et al. Relationship of sleep quality and healthrelated quality of life in adolescents according to self- and proxy ratings: a questionnaire survey. Front Psychiatry 2012;3:76. 